\title{
Analysis of Visual Language Characteristic in Logo Design
}

\author{
Xiaowei Zhang \\ Yantai Nanshan University \\ Longkou, Shandong, China \\ E-mail: 45821665@qq.com
}

\begin{abstract}
Logo develops from primeval signs, totem to present logo with modern significance that is protected by law, and it is a result of human civilization, development of commodity production and exchange. In the process of long history of the development, visual language characteristic of logo also changes.
\end{abstract}

Key words—logo; symbol visual language; identifiability

\section{INTRODUCTION}

Any form is a language or is formed by language unit. Visual language in design is to turn ideas into visual form, to embody the concept of the essence through different forms, is to turn the language and ideas into clear and vivid visual image. The form is the subject of design language's expression, when the form acts on human vision and become part of human's thing activity as symbolic elements which could be saw felt and touched, the original material forms restructures and gave birth to a specific language organizational description. Design is visual readability by the form of a variety of symbolic meaning with which people familiar. Any form or shape of the elements which has been seen by people has the nature of symbol. Symbol is a visual medium, conveying all sorts of things, phenomenon, content to the human, it has a conventional means. In the field of art, $\log 0$ with its high generalization and symbolic design techniques, concise and strong graphic symbols, accurate information demands, a unique style. This paper is a summary of the main features of the visual language of the contemporary logo design.

\section{EXPRESSION WITH HIGH DEGREE OF ALL- INCLUSIVENESS AND IDENTIFIABILITY}

\section{A. All-inclusiveness}

Language of logo design should be highly generalized, that means all-inclusiveness, but this generalization should not be straightforward and simple, it is a expression of essential characteristics of things after concentration with accurate, clear, appropriate visual language. According to the theory of the psychology of the format tower, human's vision tends to decompose complex objects and is used to simplify and simplify the nature. By the sense of the meaning of communication to simplify the meaning of the structure system and present the meaning of the style to achieve the "identity", that is, the rich meaning and diversity of the form of organization in a unified structure. The concept of "identity" is an accurate annotation of the visual language of the visual language of logo design. Information dissemination is very important in modern society. Through a thorough analysis of things and accurate judgment, find out the essentials of things, carefully refined, accurate information to understand the connotation, concise beauty of visual language, is suitable and appropriate visual image of the language expresses the design of the main body and psychic factors.For example: Image of the General Company logo, just the first two letters of GENERALMOTORS, "GM", it has fully explained the essence. Actually, the General Company as the world's largest car company doesn't need gild the lily. In addition, GM's production of multipurpose luxury car "Chevrolet", not only the text logo is named after racing Chevrolet name, its logo is the abstraction of butterfly knot, as abstract painter's masterpiece, the logo is concise and lively, extraordinary refined. As for the company's production of "Saturn" brand cars, its text is from the United States "Saturn" moon probe satellite, the graphic symbols, looks like the orbit of "Saturn to the Moon". Although it is very simple, it contains rich content of scientific, technological, modern and future. Generally speaking, the more abstract and inclusive, the more extensive and profound reflection of the objective things is.

\section{B. Identifiability}

The requirement of Logo design is to create a unique identification symbol which pursues concise. If the content of the theme is very exhaustive, it will lead to the complex, cumbersome of visual elements and get the opposite effect. So it is necessary to adopt accurate ideas and creative forms to attract audience's attention through unconventional style, concise, vivid color to strengthen symbol recognition and memory. If we can design a mature logo with a distinctive feature, people can easily identify, and difficult to forget. The memory of the logo design has important role, it can make the viewer extract the sign information from the brain in the purchase behavior, purchase commodity according to the sign. For example: Japan Dairong department store logo adopts first letter "d" of its English name and morning sun image combination as the main graphics, it is a concise style. The striking orange, easy identified font which further 
strengthens symbol recognition, has a strong visual impact, not easy to forget.

\section{RICH IMAGINATION AND IMAGERY DESCRIPTION}

\section{A. Imagination}

Logo is one kind of symbolic art, is one kind of symbol, symbol itself is one abstract concept. Logo, as the form of the visual language, is given a variety of symbolic meaning. Symbol is composed by form graph with certain meaning and pattern, it represents the purpose, content, nature, characteristics, ideas, spirit. Logo design is to abstract things to show with visible or graphic form. Human's understanding of things has also experienced a process from the perception of the form to the overall structure of the form to understand (point, line, surface, color, texture, etc.). People can not only recall the image of the past, but also create a new image. People used imagination of old experience (that is, the old appearance) to re mix, resulting in a new image of things. Imagination is a kind of psychological process, which produces a new image from the original image in people's mind and it is a kind of advanced complex cognitive activity. Figurativeness and novelty are the basic features of the imagination, which mainly deal with the graphic information in people's minds, rather than words, symbols, concepts and so on.

\section{B. Imagery description}

Logo design is the transformation process from the abstract meaning to the concrete expression, so imagery description is very important. The basis of Imagery description is "image", it is the visual image, but "consciousness", that is the subjective, combination of the two can constitute the so-called "imagery description ". According to the theory of visual perception, the image should include the interaction of several aspects: first is the objective visible part (Vision), the second is the imaginary part (Imagination), and the last is "Composition" part. As the visual image of imagery description, due to the direct perception and memory of the visual object, the subject and object of direct exchange has obvious intuitive features and turn the object of the image through the visual into the idea, the subject of emotion, experience and personality and so the image can be born to express and eventually completed. Obviously, the visual image is an image from the object to the intermediary. Visual object is a process of consciousness, and it is a process of "natural person" which is fully involved in the process of human's consciousness. Logo design, form of the visual language, need through rich imagination to people's visual experience basis from the object to image expression. For example: Mengniu Dairy (Group) Co., Ltd. logo, it is designed to determine the geographical location of the environment and the characteristics of the image of the characteristics of the cow's characteristics, product characteristics, corporate culture, corporate spirit, the pursuit of goals and other abstract concepts. Mengniu enterprises located in Inner Mongolia, in our impression, it is the blue sky, white clouds, the vast grassland, flocks of cattle and sheep, aso Mengniu is mainly engaged in the production of milk products, "niu" also means bull market or bullish. According to these two characteristics, the design patterns of the logo are full of rich imagination and the rich connotation of the image, which is expressed by the simple symbol image. The symbol, similar circular pattern on behalf of the horn, represent a cow, the graphics like a crescent shaped, is a symbol of Islam "crescent Han Star". Mengniu products are Islamic food. Under the horn, it is a stroke pattern and an image of the open prairie, the whole logo graphic image language describes the Mengniu enterprises unlimited running life vigor and enterprising spirit.

\section{PROFOUND CULTURAL CONNOTATION AND EXQUISITE STYLE DESIGN PERFORMANCE}

\section{A. Cultural Connotation}

The success of a logo must be tested by practice. To make the mark spread far, only the surface of the form of aesthetic feeling is difficult to complete。 The logo must have a profound connotation so that the audience will have a relevant association. Emotional communication will make the logo has a long life. So the successful logo design is a logo with thought, soul, vitality. For example: the Chinese bank's logo is designed by the famous designer Jin Daiqiang. He combined ancient coin and character "Zhong" to express "Chinese heart", which especially reflects the characteristics of Chinese. From the point of view of the constitution of the overall pattern, coin shape represents the characteristics of bank industry, the word "zhong" symbols of China, outer circle implies that Bank of China is a global international bank.

\section{B. Pattern Design}

Logo design should be clever, beautiful shaped, good looking and easy to be remembered. The pattern of logo design needs to have a certain degree of typicality and a wide range of recognition. For example: in 2008, the Olympic Science Conference logo, the emblem of the yellow, red, black, green, the four arcs representing the four of the general assembly of the international organization. Yellow and blue two curves constitute the letter "S", representing "science" and "Sport" sports. At the same time, the vitality of the yellow and blue, respectively, representing sports and science, it also reflects the theme of the Olympic Science conference. "Auspicious clouds" is always Chinese nation's thriving and prosperous symbol. The composition of the above elements, look like a blooming flower, while the rotation curve is a symbol of eternal power of sports and science.

\section{CONTEMPORANEITY AND NATIONAL CHARACTER}

\section{A. Contemporaneity}

History is continuing, the time is developing, and the society is also unceasingly progressing. Logo design patterns are also in the process of constant change and innovation. In particular, the current information communication is convenient and international exchanges are more frequent. Because of the mutual influence of the exchange, the visual 
language of the form of the symbol is gradually converging to the world's common language of art. At present, time sense of the form design of logo presents a concise, profound and innovative and attractive, freedom, integrity of the beauty of the design. In the global economy of today, the sense of the Times is to enable the design keep abreast with the pace of the times, keep pace with the world and to allow more people to understand and recognize so that they can resonate. For example: Coca-Cola's logo (CoCaCala), which is based on the text of the English language has become the main international symbol. In today's world economic integration, text logo has become an indispensable part of many enterprises and the logo of the pure text holds more and more great proportion in the design. Many famous companies are adopting text as a company logo. Over half of the world's top ten famous enterprises such as 3Mand IBM adopt the text labels. The value of the brand constantly improves in the process of its development, and its visual image also changed, so the design of the logo is not invariable. Even it is a successful logo, it will change and adjusts properly in the original basis with the times as people's aesthetic needs changes. For example, the first Apple logo is very complex, it is a pattern in which Newton was sitting under the apple tree reading a book, the up and down is a winding ribbon, writing the word Apple Computer Co. The frame quoted British poet William - Wordsworth, William Wordsworth's short poems: "Newton, a soul forever lonely sailing in strange seas of though." The company's responsible person later considered that this logo was too complicated and has negative effect on sales, so the company hired McKenna Regis Consultants Co's Rob (Janoff Rob) to design a new logo for apple. This is Apple's second logo, a round of the rainbow apple logo. Between 1976 and 1999, Apple has been using this logo. Later evolved into the current new Apple logo, bitten apple, "bite" in English has same pronunciation with "byte" which is computer's basic operation unit.

\section{B. National Character}

National character is to make the design unique with closeness. Increasing trend of single language convergence will obliterate the personality. Absence of difference also weakens the identifiability, people's memory will not last long as s result. From my view, contemporaneity focus on external aspects and national character should be connotations of the design language based on cultural and artistic conception. For example: Beijing 2008 Olympic Games logo design, it is widely accepted and praised by experts and the general public. The form of the logo is smooth and filled rich sense of sport, lively and free spirited. The visual language is clear and stylish, which reflects distinct contemporaneity and more important a wealth of traditional culture and the spirit of the Chinese nation. The logo uses China traditional handicrafts "concentric knot", also called "China knot" of pattern styling, "knot" means reunion, closeness and best wishes, plus "knot" of "China knot" in Chinese pronounces "jie", it is similar with "JI" and "JI" has rich meaning, such as fortune, emolument, longevity, happiness, safety and health. Therefore, the "knot" as folk art with vitality symbols the quintessence of Chinese culture, flourished and spread and become one of the symbols of China. In addition, "Chinese knot" pattern shows dynamic gesture of a person who is practicing Tai Chi, Tai Chi is also a symbol image of China, the philosophy of taiji is Chinese traditional Taoism, it absorbed the essence of "qigong" and kung fu, it also has a close relationship with Confucianism and Buddhism, demonstrating the philosophy of the Chinese ancient wisdom. Tai Chi humanoid form expresses beauty, harmony, and strength. It also means the cooperation and culture exchanges among people of the world

\section{CONCLUSION}

Logo design plays an important role in our social life and it also plays an important role in the field of art design. Logo design develops continuously in mutual exchanges, the visual language of modern logo design patterns also changes and produce new ideas, the analysis of the same can enable us to firmly grasp the core semantic value design domain with rich content, changeable graphic style and active idea. By this way, we can design more works, which is full of wisdom and imagination and is consistent with the logo artistic characteristics, easy to identify and remember.

\section{REFERENCES}

[1] Wang Shouzhi. Modern Design History of the World, New Century Press, 1995

[2] Zhaochangzhisi, Lv Qingfu, Art design of planar formation, the people's press, 1986

[3] Qiu Chengde. Shaanxi people's fine arts publishing house, 1997

[4] Guo Zhenshan. Logo design, Hunan University press, 2010 